\title{
The Animated Database Courseware (ADbC) and the Database Design Module
}

\author{
Mario A.M. Guimaraes, Meg Murray \\ Department of Computer Science and Information Systems \\ Kennesaw State University \\ Kennesaw, GA, 30144 \\ mguimara@kennesaw.edu,mcmurray@zt.kennesaw.edu
}

\begin{abstract}
There is a need to extend the breadth and depth of database curricula and to find ways to incorporate newer technologies [1]. One way to address this challenge is through supplementary instructional materials [2]. However, very few supporting materials exist that aid in the teaching of teach database concepts $[3,4,5]$. This was the motivation for constructing the Animated Database Courseware (ADbC) that is funded by NSF Grant \#0717707. ADbC has four main modules: database design, SQL, database transactions and database security. The software has a low learning curve. It has been made freely available and is located on the Internet at http://adbc.kennesaw.edu. This demonstration focuses on the Database Design Module.
\end{abstract}

Keywords: Courseware, Database, Normalization, ER Diagrams, SQL, Transactions, Security

\section{Introduction}

Database technology is a core knowledge area in computing curriculum. A good database is predicated on good database design. Database design involves the conceptualization of an underlying data model and determining an appropriate structure to be utilized in the physical implementation of that model. The conceptual and abstract nature of database design makes it inherently challenging to teach and learn. Interactive instructional materials can be effective as supplemental classroom instruction. This tutorial demonstrates a set of software animations developed to support the teaching of database design. The animations are fairly intuitive to use and are independent of any specific database text or product. The animations are part of a larger animated database courseware project funded through a National Science Foundation Course, Curriculum and Laboratory Improvement grant. The courseware is freely available and may be accessed at http://adbc.kennesaw.edu. The database design module focuses on the relational database model.

\section{The Database Design Module}

The database design module consists of seven sub-modules that progress through various database design concepts and processes. The first sub-module, ER Notations, allows 
users to visualize the same ER diagram presented in several different notations sets and compare them side-by-side (Figure 1). Scenarios to ER displays a scenario and asks users to identify the ER Diagram which best depicts the information provided (Figure 2). In ER to Tables, a user is given an ER Diagram and asked to choose the best solution for mapping the diagram to a set of relational database tables (Figure 3). In Functional Dependencies, users identify functional dependencies given a specific scenario. In Normalization, users must normalize the tables given a set of functional dependencies (Figure 4). In Denormalization, users are asked to identify the best scenarios for denormalizing. In Database Anomalies, users are asked to insert, update and delete in both normalized and unnormalized tables.

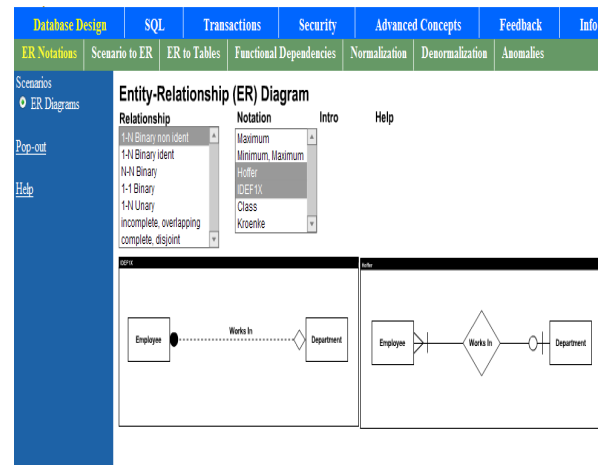

Figure 1 - ER Notations

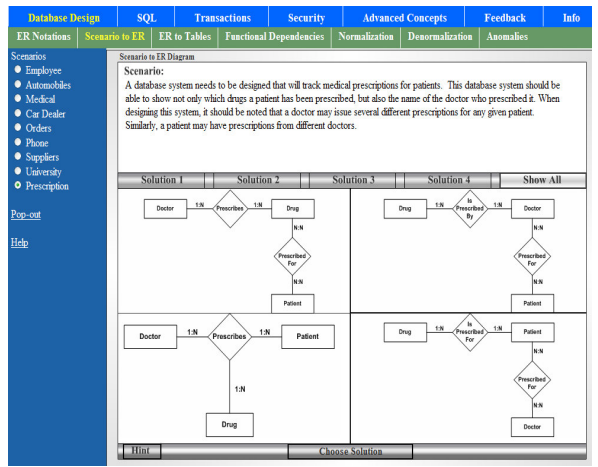

Figure 2 - Scenarios to ER

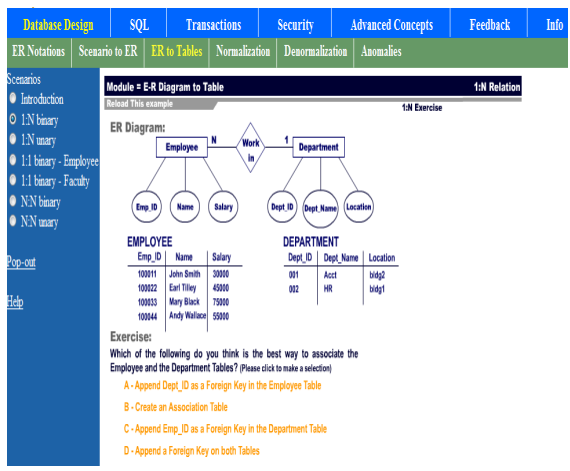

Figure 3 - ER to Tables

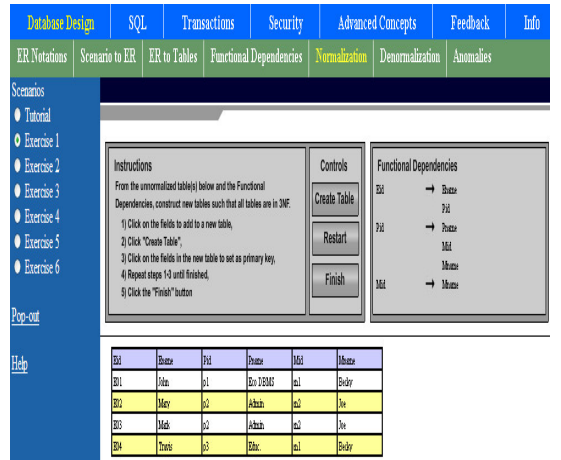

Figure 4 - Normalization

\section{Additional Modules}

\subsection{SQL}

The SQL module consists of five sub-modules. Interactive SQL allow users to construct through drop down menus simple DDL and DML operation, simple and advanced SQL queries, and Views. Pseudocode allows users to animate a query in a step by step 
format as well as see the corresponding Relational Algebra and Procedural code solutions (Figure 5). Embedded SQL allow users to compare two different solutions for accessing database from within a programming environment such as Java, C++ and PL/SQL. Stored Procedures, Functions and Triggers display how to use them as well as their purpose. Referential Integrity displays what happens to Delete and Update commands in a Parent table when there are related records in a child table.

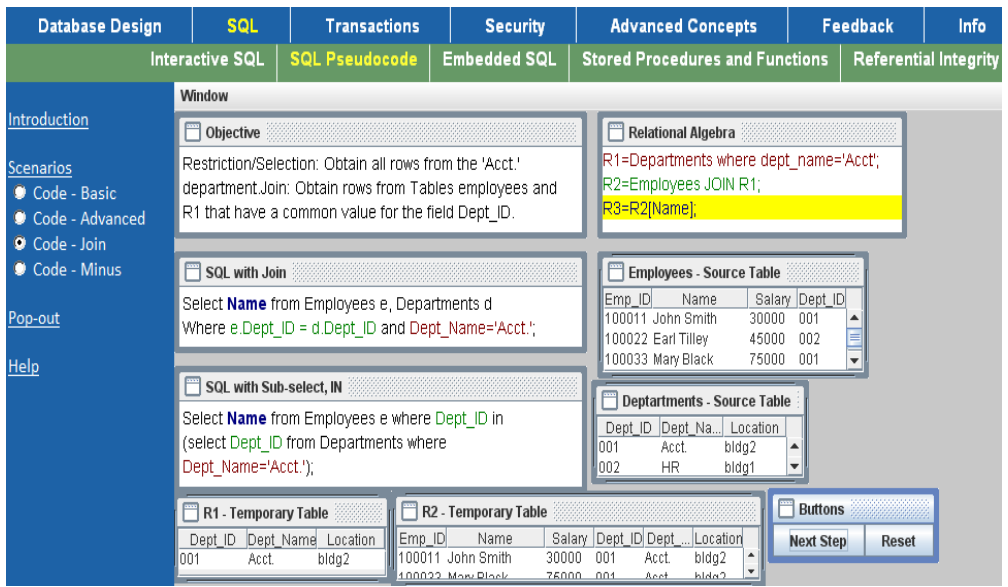

Figure 5 - Multiple SQL windows and Relational Algebra

\subsection{Transactions}

The Transactions module consists of three sub-modules. Concurrency demonstrates the various locking techniques available that handle concurrency problems in multi-user databases (Figure 6). Recovery demonstrates the difference between the logical and physical updates. For the administration sub-module, Backup and Recovery as well as Startup and Shutdown animations are also being constructed to illustrate what happens with these operations that are executed on a frequent basis.

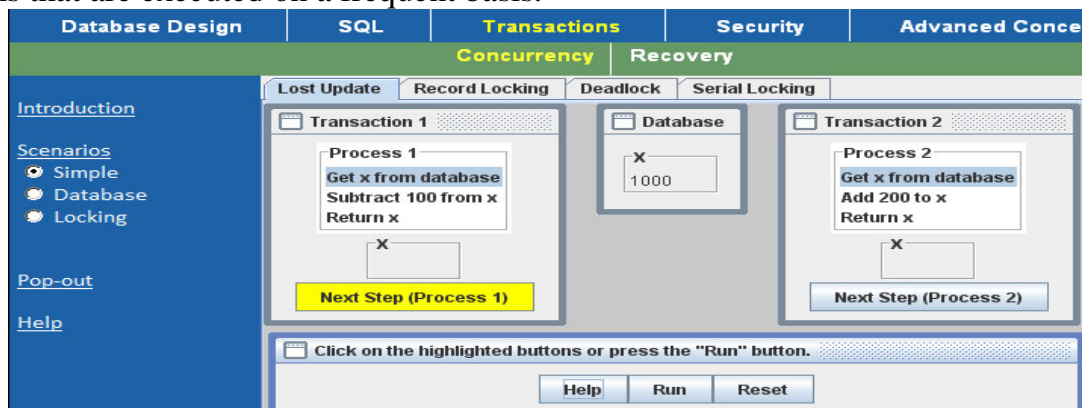

Figure 6 - Concurrency Example

\subsection{Security}

The Security module consists of six sub-modules. Access controls displays the various options of the GRANT and REVOKE command. A security matrix identifies actions 
taken on database tables via form input or report output. Row-level security implements Virtual Private Databases through views. SQL injection provides a walk-through of one of the most common exploits of the SQL injection as well as recommendations to combat it. Database Inference depicts how data may be inadvertently obtained from a database as a result of a user's ability to make inferences. Database Auditing depicts how triggers can be useful in database auditing.

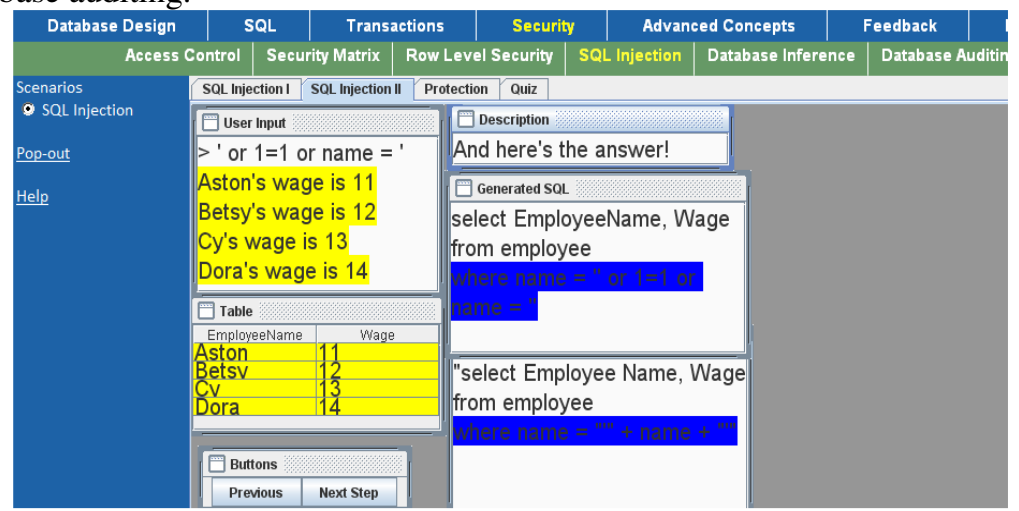

Figure 7 - SQL Injection

\section{Conclusions}

The ADbC courseware project was motivated by the need to expand the database curriculum and the lack of supplementary materials. This courseware has over 100 exercises and animations that cover a wide range of database topics. The animations are fairly intuitive to use and independent of any specific database text or product. As we expand the courseware and conduct usability tests, we will also publish new ways to incorporate this product into the classroom.

\section{References}

[1] Adams, E. S., Granger, M., Goelman, D. and Ricardo, C. Managing the introductory database course: what goes in and what comes out? Proceedings of the $35^{\text {th }}$ SIGSCE technical symposium on Computer Science education, 497-498, 2004.

[2] Naps, T.L.,et.al. 2002. Exploring the role of visualization and engagement in computer science education. In Working Group Reports From ITiCSE (Aarhus, Denmark, June 24-28, 2002). ITiCSE02. ACM-Press, NY,NY,131-152. DOI= http://doi.acm.org/10.1145/960568.782998

[3] w3schools, Full web building tutorials, http://www.w3schools.com/

[4] Dietrich, S., Urban, S., WinRDBI Educational Tool, ehttp://winrdbi.asu.edu/

[5] Dietrich,S., Urban,S., Beyond Relational Databases, http://www.eas.asu.edu/ advdb/ 